\title{
Metagenomic analysis on resistance genes in water and microplastics from a mariculture system
}

\author{
Jian Lu ${ }^{1,3}$, Jun Wu $(\bowtie)^{2}$, Jianhua Wang ${ }^{1}$ \\ 1 CAS Key Laboratory of Coastal Environmental Processes and Ecological Remediation, Yantai Institute of Coastal Zone Research (YIC), Chinese \\ Academy of Sciences (CAS); Shandong Key Laboratory of Coastal Environmental Processes, YICCAS, Yantai 264003, China \\ 2 Yantai Research Institute, Harbin Engineering University, Yantai 264006, China \\ 3 Center for Ocean Mega-Science, Chinese Academy of Sciences, Qingdao 266071, China
}

\section{H I G H L I G H T S}

- Total 174 subtypes of ARGs were detected by metagenomic analysis.

- Chloramphenicol resistance genes were the dominant ARGs in water and microplastics.

- The abundances of MRGs were much higher than those of ARGs.

- Proteobacteria, Bacteroidetes, and Actinobacteria were the dominant phylum.

- Microplastics in mariculture system could enrich most of MRGs and some ARGs.

\section{A R T I C L E I N F O}

\section{Article history:}

Received 14 May 2021

Revised 21 June 2021

Accepted 25 July 2021

Available online 8 September 2021

\section{Keywords:}

Antibiotic resistance genes

Metal resistance genes

Metagenomic analysis

Microplastics

Mariculture

\section{GR A P HIC A B T R A C T}

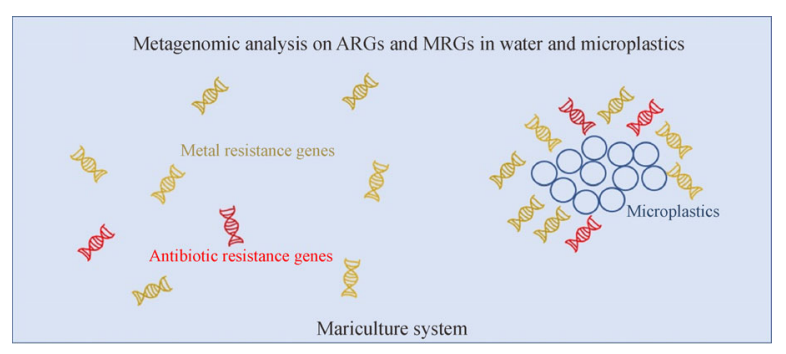

\section{A B S T R A C T}

Microplastics existing widely in different matrices have been regarded as a reservoir for emerging contaminants. Mariculture systems have been observed to host microplastics and antibiotic resistance genes (ARGs). However, more information on proliferation of ARGs and metal resistance genes (MRGs) in mariculture system at the presence of microplastics is needed. This study used metagenomic analysis to investigate the distribution of ARGs and MRGs in water and microplastics of a typical mariculture pond. Total 18 types including 174 subtypes of ARGs were detected with the total relative abundances of $1.22 / 1.25$ copies per $16 \mathrm{~S}$ rRNA copy for microplastics/water. Chloramphenicol resistance genes were the dominant ARGs with the abundance of $0.35 / 0.42$ copies per $16 \mathrm{~S}$ rRNA copy for microplastics/water. Intergron intI1 was dominant gene among 6 detected mobile genetic elements (MGEs) with the abundance of $75.46 / 68.70$ copies per $16 \mathrm{~S}$ rRNA copy for water/microplastics. Total 9 types including 46 subtypes of MRGs were detected with total abundance of $5.02 \times 10^{2} / 6.39 \times 10^{2}$ copies per 16S rRNA copy for water/ microplastics while genes resistant to copper and iron served as the dominant MRGs. Proteobacteria, Bacteroidetes, and Actinobacteria accounted for $84.2 \% / 89.5 \%$ of total microbial community in microplastics/water. ARGs with relatively high abundance were significantly positively related to major genera, MGEs, and MRGs. Microplastics in mariculture system could enrich most of MRGs and some ARGs to serve as potential reservoir for these pollutants. The findings of this study will provide important information on resistance gene pollution at presence of microplastics in the mariculture system for further proposing suitable strategy of environmental management.

\section{Introduction}

Microplastics, known as plastic particles with size less than $5 \mathrm{~mm}$, have been determined as emerging contaminants to

$\triangle$ Corresponding author

E-mail: wujunlisa@163.com

Special Issue-Microplastic and Nanoplastic Pollution: Characterization, Transport, Fate, and Remediation Strategies (Responsible Editors: Wen Zhang, Melissa Pasquinelli \& Yang Li) attract worldwide attentions (Al-Salem et al., 2020). Microplastics ubiquitously exist in aquatic systems (Elizalde-Velázquez and Gómez-Oliván, 2021), soils/ sediments (Koutnik et al., 2021), air (Wright et al., 2020), and organisms (Santana-Viera et al., 2021). Microplastics have been regarded as the important hotspots for many pollutants such as persistent organic pollutants (Santana-Viera et al., 2021), endocrine disrupting contaminants ( $\mathrm{Lu}$ et al., 2021a), and emerging contaminants (Lu et al., 2019a). Microplastics have important effect on pollutant sorption and release, toxicity of multiple systems, 
and the organisms in the environment to exert potential risks to human health and ecological safety (Liu and Wang, 2020; Prata et al., 2021; Xu and Ren, 2021). Ocean is an important sink to receive plastic debris with annual amount of 4.8-12.7 million tons (Al-Salem et al., 2020). Anthropogenic generation rate of microplastics is estimated as $0.1-5500 \mathrm{~kg} / \mathrm{d}$ according to beach and shoreline monitoring (Al-Salem et al., 2020). Thus, an enormous pressure has been exerted to the marine ecosystem by microplastics.

Antibiotic resistance genes (ARGs) might be a specific and critical kind of emerging contaminants hosted by microplastics due to their potential threat to the human health and ecological sustainability. ARGs can proliferate in diverse matrices including microplastics ( $\mathrm{Lu}$ et al., 2019a, 2020a, 2020b). ARGs generally propagate in the environment through vertical gene transfer by transmitting genetic information from mother to daughter cells and horizonal gene transfer (HGT) under mediation by mobile genetic elements (MGEs) such as transposons, plasmids, bacteriophages, and intergrons (Pazda et al., 2019; Wu et al., 2020). ARGs can be induced under the natural conditions while they also proliferate under the coselective pressure from antibiotics, heavy metals, or other agents (Li et al., 2020). Propagation and dissemination of ARGs will enhance antibiotic resistance and induce antibiotic-resistant bacteria (ARB) to make a significantly negative effect on controlling the bacterial infection and epidemics (Pazda et al., 2019). The costs for hospitalcaring patients infected by ARB or multi-drug resistance bacteria have reached 2.2 billion USD in the United States and 1.5 billion EUR in the Europe (Pazda et al., 2019). Enormous risks posed by ARGs have ignited worldwide attention so that lots of research works have focused on occurrence of ARGs in different matrices (Li et al., 2020; Lu et al., 2019b; Pazda et al., 2019). ARGs have been observed to proliferate and accumulate on microplastics in different environments (Lu et al., 2019a; Pham et al., 2021; Yang et al., 2019). Wide distribution of microplastics might further enhance the spread of ARGs and ARB to deteriorate the pollution status. Most of studies on ARGs related to microplastics still adopt qPCR methods to obtain the incomplete information for assessment. Therefore, metagenomic analysis which takes a wide range of ARGs into consideration (Nowrotek et al., 2019; Wang et al., 2018; Yin et al., 2018, 2019) might be more useful and thorough for investigating ARGs on microplastics.

Heavy metals have been widely detected in various matrices (Lu et al., 2021b) so that metal resistance genes (MRGs) have been induced to exist in different environments (Sherpa et al., 2020; Yang et al., 2019). Organic pollution is also reported to induce proliferation of MRGs (Sherpa et al., 2020). Co-occurrence of ARGs and MRGs have been widely observed (Sherpa et al., 2020; Yang et al., 2019) to comprehensively represent the resistance gene pollution status.

Mariculture is one essential industry in the coastal zone for economic development ( $\mathrm{Lu}$ et al., 2021c). Metagenomic analysis is a useful and efficient tool for determining ARGs, MGEs, MRGs, and microbial community simultaneously. Metagenomic analysis have been used to investigate the distribution of ARGs and MRGs in the environment (Sherpa et al., 2020; Yang et al., 2019) with wider viewpoint. Therefore, this study first used metagenomic analysis to explore the distribution of ARGs, MGEs, MRGs, and microbial community in water and microplastics of a typical mariculture system. The objectives of this study are to provide the comprehensive information on ARGs and MRGs in mariculture system containing microplastics and discuss the potential correlation among ARGs, MGEs, MRGs, and microbial community. The findings of this study will provide some insights on environmental management of mariculture.

\section{Materials and methods}

\subsection{Sampling, DNA extraction, and metagenomic sequencing}

One water sample with volume of $2 \mathrm{~L}$ was collected from a shrimp pond with volume of $1000 \mathrm{~m}^{3}$ of a recirculating mariculture system near Dongying City and passed through $0.22-\mu \mathrm{m}$ mixed cellulose esters filters (Millipore, Germany). One microplastic sample was in situ obtained from the same pond by passing $100-\mathrm{L}$ seawater collected from the different positions of the pond and mixed evenly through a $2 \mathrm{~m} \times 2 \mathrm{~m}$ net with $300-\mu \mathrm{m}$ mesh size. The samples were put into a sterilized container with icebox and transported to the laboratory as soon as possible. The abundance of microplastics was determined as 261 items $/ \mathrm{m}^{3}$ by counting method. The type of microplastics was determined by Fourier transform infrared spectroscopy as polyethylene terephthalate (PET), similar to the previous study (Lu et al., 2019a).

TIANamp Soil DNA Kit (Beijing, China) was used to extract total DNA of water $(2 \mathrm{~L})$ and microplastic (collected from 100-L seawater) samples based on the guideline of the manufacturer. Agarose gel (1\%) electrophoresis was used to identify the purity of extracted DNA while NanoDrop UV-vis spectrophotometer (Thermo Scientific, Wilmington, USA) was adopted to determine the DNA concentration.

Illumina Novaseq6000 platform (San Diego, CA, USA) was used to construct and sequence the short-gun metagenomic library by Majorbio company (Shanghai, China). The sequences with length $<20 \mathrm{bp}$ were removed by Fastp (Version 0.20.0). The clean base for water/ microplastic sample was $1.73 \times 10^{10} / 1.55 \times 10^{10}$ bp to account for $99.4 \% / 99.1 \%$ of its raw reads. The raw sequences of water and microplastic samples were deposited into NCBI database with the accession number of PRJNA728751. 


\subsection{Metagenomic quantification of target genes}

BLAST (Version 2.2.28) was used to align the sequences against NCBI NR (non-redundant protein sequence) database with e-value threshold of $1 \times 10^{-5}$ for taxonomy annotation.

The filtered reads were input into ARGs-OAP v2.0 online pipeline to annotate ARGs (Yin et al., 2018) with evalue of $10^{-7}$ and similarity higher than $80 \%$. Total 24 types of ARGs were investigated by ARGs-OAP platform. The abundance of ARGs were normalized with unit of ARGs copies per 16S rRNA copy (Yin et al., 2018) to make the abundance data comparable with the previous studies.

Intergrons serving as representative MGEs were investigated. The filtered reads were aligned using BLAST software with INTEGRALL database with e-value of $10^{-5}$ and similarity higher than $90 \%$. The abundances of MGEs were further normalized by $16 \mathrm{~S}$ rRNA copies which were obtained from ARGs-OAP pipeline.

BacMet 2.0, a metal resistance gene (MRG) database was used for MRG annotation. The filtered gene-like reads were aligned with length greater than 25 amino acids, evalue of $10^{-5}$, and identity higher than $90 \%$. The abundances of MRGs were also normalized by $16 \mathrm{~S}$ rRNA copies.

\subsection{Visualization and statistical analysis}

Heatmap and column diagrams of target genes were prepared by OriginPro 2019 (OriginLab, USA). Correlation analysis with significance at $p<0.05$ was performed by SPSS 19 (IBM, USA). Circos analysis was conducted by Circos-0.67-7. Network among the target genes and bacteria was generated by Cytoscape 3.7.2 (The Cytoscape Consoritum, USA) with absolute value of Spearman coefficient greater than 0.8 and $p<0.05$.

\section{Results}

3.1 Occurrence of ARGs on microplastics in mariculture environment

Total 18 types including 174 sub-types of ARGs were detected in the samples (Fig. 1). Genes resistant to carbomycin, fusaric acid, fusidic acid, puromycin, spectinomycin, and tetracenomycin $\mathrm{C}$ were not detected in all samples while those resistant to fosfomycin/polymyxin were not detected in the microplastic/water sample. Normalized by $16 \mathrm{~S}$ rRNA copies, the relative abundance of chloramphenicol resistance genes was higher than that of the remaining ARG types to reach 0.35 and 0.42 copies/ 16S rRNA copy for microplastics and water sample, respectively (Fig. 1). The relative abundances of the remaining detected ARGs were in the range of $1.57 \times$ $10^{-5}-2.78 \times 10^{-1}$ copies/16S rRNA copy for microplastics and $4.89 \times 10^{-5}-3.47 \times 10^{-1}$ copies/16S rRNA copy for water. The relative abundances of total detected ARGs reached 1.22 copies/16S rRNA copy for microplastics and (a)
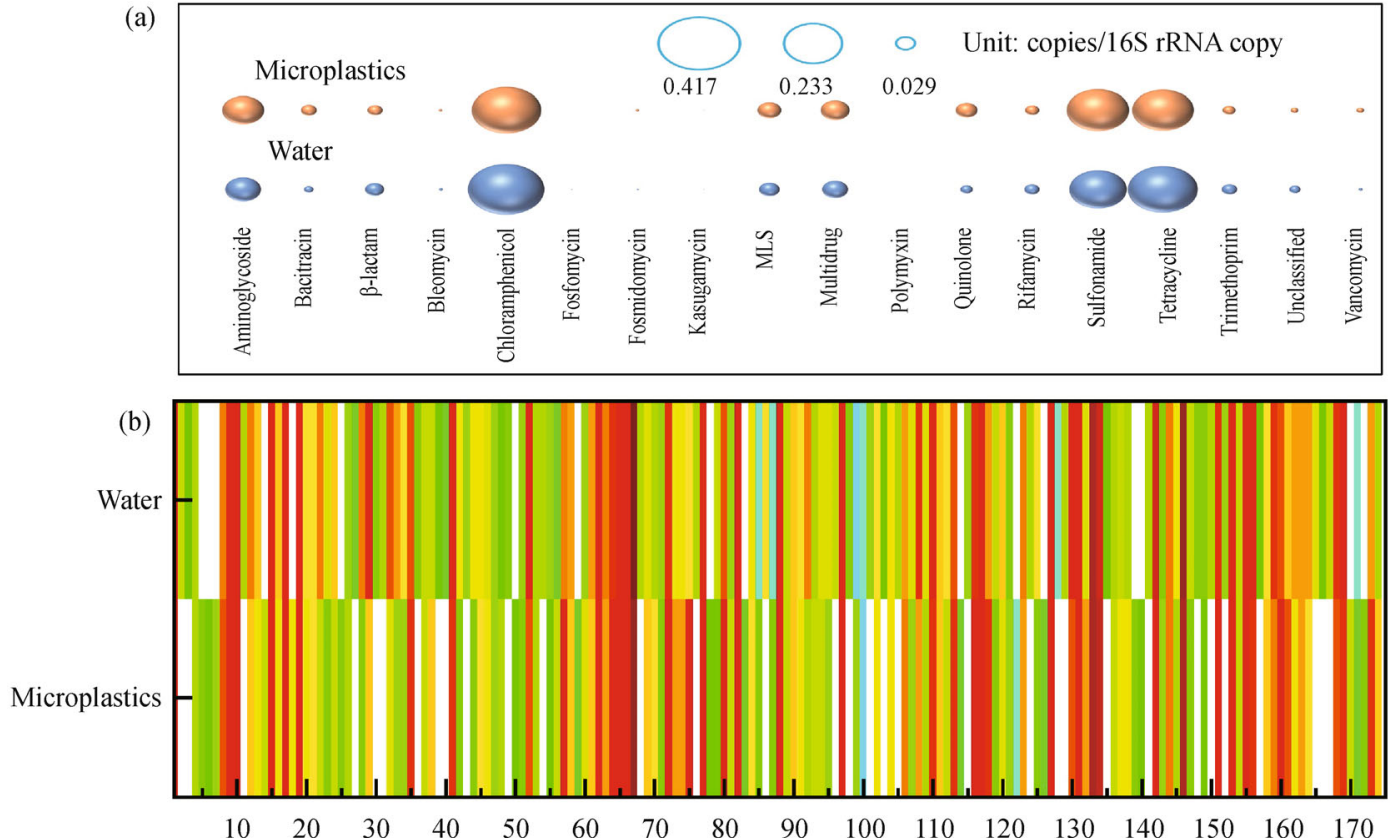

Relative abundance (copies/16S rRNA copy)

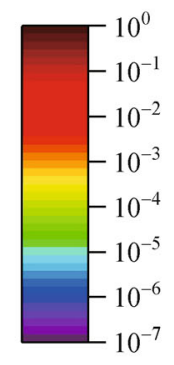

Fig. 1 The relative abundances of different types (a) and subtypes (b) of detected ARGs in water and microplastic samples (Detailed subtypes corresponding to individual digit of $x$-axis in Fig. 1-(b) referred to Table S1). 
1.25 copies/16S rRNA copy for water. The abundance of top 9 ARG types followed the order of chloramphenicol $>$ sulfonamide $>$ tetracycline $>$ aminoglycoside $>$ multidrug $>$ macrolide-lincosamide-streptogramin $>$ quinolone $>$ bacitracin $>\beta$-lactam for microplastic sample and chloramphenicol $>$ tetracycline $>$ sulfonamide $>$ aminoglycoside $>$ multidrug $>$ macrolide-lincosamide-streptogra$\min >\beta$-lactam $>$ trimethoprim $>$ rifamycin for water sample (Fig. 1). The relative abundances of ARGs in mariculture system were significantly higher than those in marine system in terms of water and microplastic samples (Yang et al., 2019), suggesting that mariculture might be the important contributor for ARGs in marine environment.

Approximately 141 sub-types of ARGs were detected in microplastics while 157 subtypes were detected in water. Genes including $f l o \mathrm{R}$, sul 1 , and tet $\mathrm{G}$ were the predominant ARG subtypes to account for $51.72 \%$ of total ARGs for microplastics and $59.73 \%$ for water in terms of abundance normalized by $16 \mathrm{~S}$ rRNA gene (Fig. 1). Gene $f l o \mathrm{R}$ was the dominant chloramphenicol resistance gene with the relative abundance of $2.88 \times 10^{-1}$ copies/16S rRNA copy for microplastics and $3.60 \times 10^{-1}$ copies/16S rRNA copy for water while van R was the dominant vancomycin resistance gene with the relative abundance of $3.15 \times 10^{-3}$ copies/16S rRNA copy for microplastics and $1.05 \times 10^{-3}$ copies/16S rRNA copy for water. Gene sul1 was the dominant sulfonamide resistance gene with the relative abundance of $1.84 \times 10^{-1}$ copies/16S rRNA copy for microplastics and $1.51 \times 10^{-1}$ copies/16S rRNA copy for water while $m f p \mathrm{~A} /$ tet $\mathrm{G}$ was the dominant quinolone/ tetracycline resistance gene with the relative abundance of $3.09 \times 10^{-2} / 1.59 \times 10^{-1}$ copies/16S rRNA copy for microplastics and $6.37 \times 10^{-3} / 2.36 \times 10^{-1}$ copies/16S rRNA copy. Gene bacA was the dominant bacitracin resistance gene subtype with the relative abundance of $1.76 \times 10^{-2}$ for microplastics and $5.86 \times 10^{-3}$ copies $/ 16 \mathrm{~S}$ rRNA copy for water while $\operatorname{ros} \mathrm{A}$ was the major fosmidomycin resistance gene with the relative abundance of $4.79 \times 10^{-4}$ for microplastics and $1.97 \times 10^{-4}$ for water. Distribution of ARG subtypes of the same type showed significant difference. Only one subtype resistant to fosfomycin/kasugamycin/polymyxin was detected in the samples while 41 subtypes of multidrug resistance genes and $39 \beta$-lactam resistance genes were detected during the investigation. The relative abundances of subtypes resistant to sulfonamide or fosmidomycin were generally similar while those of the remaining ARG subtypes showed the significant variation for both microplastics and water.

3.2 Occurrence of MGEs and MRGs on microplastics in mariculture environment

Total 6 integrase intergrons were detected in water and microplastics of mariculture system (Fig. 2(a)). Gene intI1 accounted for over $94 \%$ of detected MGEs with 75.46
copies/16S rRNA copy for water and 68.70 copies/16S rRNA copy for microplastics while that of int $I 2$ was the lowest with $7.71 \times 10^{-3}$ copies/16S rRNA copy for water and $7.17 \times 10^{-3}$ copies/16S rRNA copy for microplastics. The abundances of detected intergrons followed the order of int $11>$ groEL/intI $1>$ int $19>$ intIA $>$ orf $/$ int $I 1>$ int 12 for water and intI1 $>$ groEL/intI1 $>$ intIA $>$ orflintI1 $>$ in$t I 9>\operatorname{int} I 2$ for microplastics. Intergron int $I 1$ was related with multiple cassettes including sul1, groL, groEL, tnpA, $m r x$, ere $\mathrm{A} 2$, tet $\mathrm{A}(\mathrm{G}), \operatorname{aad} \mathrm{A} 11, \operatorname{cml} \mathrm{A} 9, \operatorname{orf} 5, \operatorname{cml} \mathrm{A}, I S C R 1$, and tet $\mathrm{R}$ according to INTEGRALL annotation while groEL/intI1 was related with cassettes including sul1, qep $\mathrm{A} 2$, tnp $\mathrm{A}$, and $c m l \mathrm{~A}$. Intergron intI9 was related with cassette orf73 while int 2 was related with cassette blaCARB-4. The relative abundances of intergrons except intIA and orflintI 1 in water were higher than those on microplastics, illustrating that mobility of ARGs in water might be stronger than that on microplastics.

The relative abundances of MRGs in both water and microplastics were much higher than those of ARGs and MGEs (Fig. 2(b)). Total 9 types of MRGs including arsenic (As), cadmium (Cd), chromium (Cr), cobalt (Co), copper $(\mathrm{Cu})$, iron $(\mathrm{Fe})$, molybdenum $(\mathrm{Mo})$, nickel $(\mathrm{Ni})$, and zinc ( $\mathrm{Zn})$ resistance genes were detected with total abundance of $5.02 \times 10^{2}$ copies/16S rRNA copy for water and $6.39 \times$ $10^{2}$ copies/16S rRNA copy for microplastics (Fig. 2(b)). The relative abundance of total MRGs was approximately 400/500 times that of total ARGs for water or microplastic sample, illustrating that metal resistance pollution might be more serious than antibiotic resistance pollution in mariculture system. Iron and copper resistance genes were the predominant MRGs to contribute to $51.95 \%$ and $35.22 \%$ of total MRG abundance for water as well as $49.52 \%$ and $34.19 \%$ for microplastics, respectively. Cadmium resistance genes among all MRGs possessed the lowest abundance of 2.57 copies/16S rRNA copy for water while chromium resistance gene showed the lowest abundance of 2.73 copies/16S rRNA copy for microplastics. The relative abundances of MRGs followed the order of iron $>$ copper $>$ molybdenum $>$ zinc $>$ nickel $>$ chromium $>$ arsenic $>$ cobalt $>$ cadmium for water and iron $>$ copper $>$ molybdenum $>$ zinc $>$ nickel $>$ cobalt $>$ cadmiu$\mathrm{m}>$ arsenic $>$ chromium for microplastics (Fig. 2(b)).

The relative abundances of total $46 \mathrm{MRG}$ subtypes were also obtained (Fig. 2(c)). The relative abundance of $y b t \mathrm{P}$ was the highest among all MRG subtypes on microplastics to reach $1.16 \times 10^{2}$ copies/16S rRNA copy while abundance of $y b t \mathrm{Q}$ was higher than that of the remaining MRG subtypes in water to reach 88.93 copies/16S rRNA copy. Total 17/11 subtypes were detected for copper/iron resistance genes. Three iron resistance genes including $y b t \mathrm{P}, y b t \mathrm{Q}$, and $y f e \mathrm{~B}$ were the predominant MRG subtypes to account for $46.44 \%$ of total MRGs in water and $43.70 \%$ in microplastics. Gene dnaK was the predominant copper resistance subtype gene for microplastics with the relative abundance of 45.98 copies/16S rRNA copy while copR 

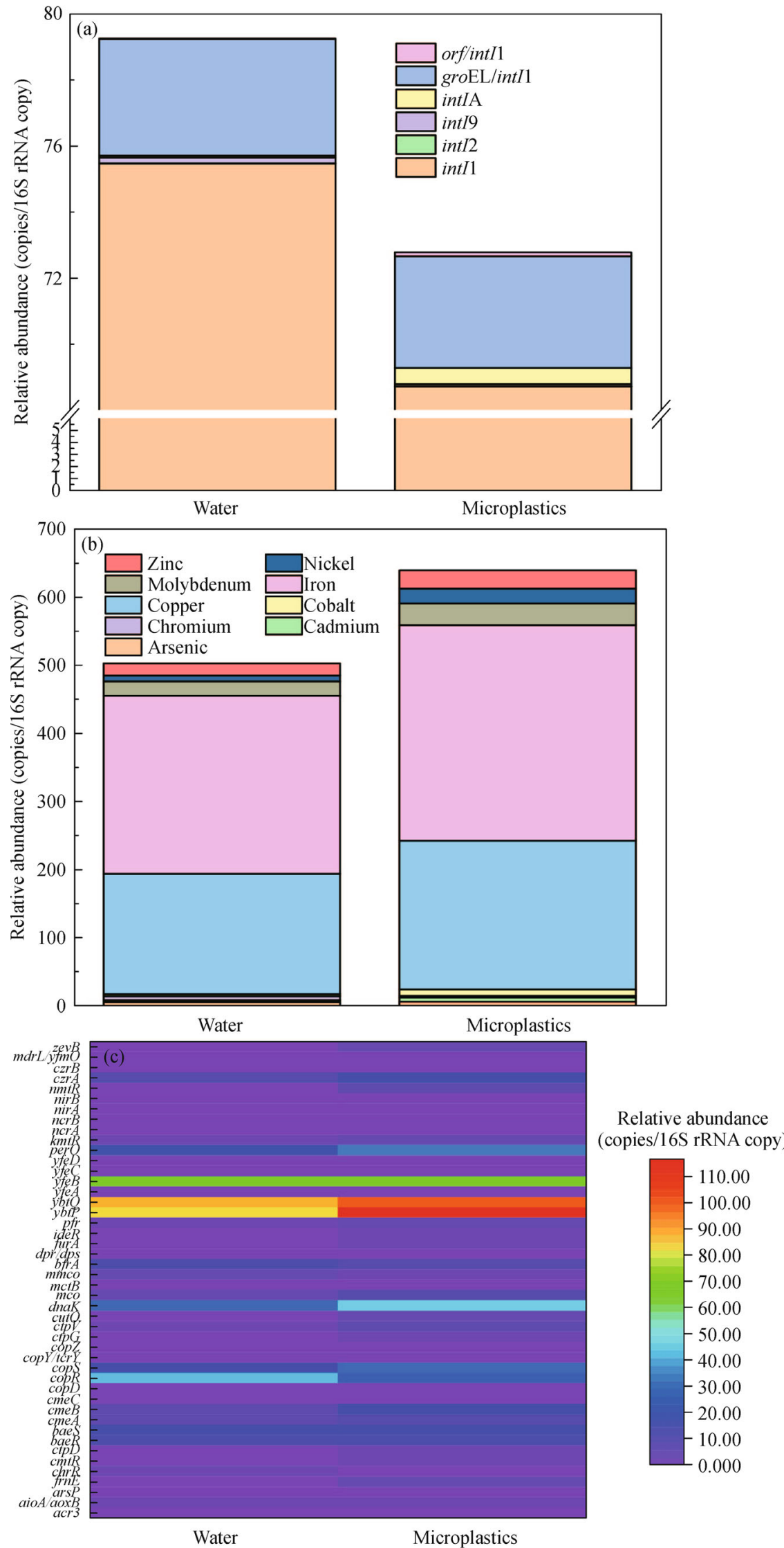

Fig. 2 The relative abundances of detected MGEs (a), MRG types (b), and MRG subtypes (c) in water and microplastic samples. 
was the dominant copper resistance gene in water with abundance of 41.01 copies/16S rRNA copy. Gene acr3 possessed the lowest abundance among all MRG subtypes for both water and microplastic samples. The ratio of $y b t \mathrm{P}$ versus acr 3 reached approximately 4100 for water and 4600 for microplastics. Gene aio $\mathrm{A} /$ aoxB with abundance of 4.14 copies/16S rRNA copy for water and 3.99 copies/ 16S rRNA copy for microplastics with possessed the highest abundance among 3 arsenic resistance genes while frn $\mathrm{E} / \operatorname{ch} r \mathrm{R} /$ perO was the only detected cadmium/chromium/molybdenum resistance gene with the relative abundance of $2.57 / 5.78 / 21.57$ copies/16S rRNA copy for water and 6.01/2.73/32.05 copies/16S rRNA copy for microplastics. The relative abundance of cobalt resistance genes including $c m t \mathrm{R}$ and $c t p \mathrm{D}$ on microplastics were 2-4 times that in water. Total 3 subtypes were detected for zinc resistance genes while $c z r$ A was the predominant gene with the relative abundance of 13.44 copies/16S rRNA copy for water and 18.07 copies/16S rRNA copy for microplastics. Total 6 subtypes were detected for nickel resistance genes while $n m t \mathrm{R}$ for microplastics with the relative abundance of 9.43 copies/16S rRNA copy and $k m t R$ for water with the relative abundance of 3.60 copies/16S RNA copy served as the predominant nickel resistance gene.

3.3 Microbial community and KEGG pathway in microplastics

Over $99 \%$ of microorganisms in microplastic and water samples were bacteria. Proteobacteria were the predominant phylum by accounting for $50.7 \%$ of microbial community for microplastic sample and $66.6 \%$ for water sample (Fig. 3(a)). Bacteroidetes/Actinobacteria/ Planctomycetes served as the second/third/forth phylum by contributing to $19.5 \% / 14.1 \% / 6.4 \%$ of microbial community for microplastics and $17.5 \% / 5.4 \% / 2.3 \%$ for water. Bacteroidetes, Actinobacteria, and Planctomycetes gradually increased in microplastics to suggest that microplastics and water provided different host environment for the microorganisms. Ruegeria was the dominant genus in water sample to account for $7.0 \%$ of total microorganisms while Mycobacterium was the dominant genus in microplastic sample to cover $10.2 \%$ of total microorganisms (Fig. 3(b)). Genera including Ruegeria, Mycobacterium, Muricauda, and Roseovarius contributed to $20.0 \%$ of total microbial community in water and $28.9 \%$ in microplastics, illustrating that microplastics might be more suitable for these to proliferate. Microbial composition in genus level showed significant difference for microplastic and water samples (Fig. 3(c)). Total 15 genera such as Mycobacterium, Muricauda, and Ruegeria exhibited significant difference with $p<1 \times 10^{-15}$ for microplastics and water. Difference in genus level for microplastics in comparison with water suggested that occurrence of microplastics might significantly change the habitat for the aquatic microorganisms.
KEGG metabolism pathway in microplastic/water sample was further investigated (Fig. 4). Amino acid metabolism, global and overview maps, and carbohydrate metabolism served as the major metabolism pathways for both microplastics and water to respectively account for over $12 \%$ of total pathways (Fig. 4(a)). The detected 15 metabolism pathways such as carbohydrate metabolism, amino acid metabolism, energy metabolism, membrane transport, and nucleotide metabolism showed significant difference between microplastics and water at $p<1 \times$ $10^{-15}$ (except replication and repair at $p<0.0005$, Fig. 4(b)), exhibiting that the existence of microplastics would have drastic effect on the metabolism pathway for molecular interaction in the mariculture environment.

3.4 Correlation between ARGs and microbial community in mariculture environment with microplastics

Bacterial genus might have different contribution to existence of ARGs in different environment. Contribution proportion of top 10 bacterial genera to 7 representative ARGs in water/microplastics was calculated (Fig. 5). Contribution proportion of total 10 predominant genera to each resistance gene ranged from $0 \%$ to $57 \%$. Existence of tet $\mathrm{G}$ in both water and microplastics was affected by the genera with relative abundance less than $1 \%$ while the 10 predominant genera for the water/microplastics did not contribute to its occurrence (Fig. 5). Muricauda was the major contributor among the predominant genera for $m a c \mathrm{~B} /$ bcr $\mathrm{A} /$ tet $\mathrm{B}(\mathrm{P})$ with the proportion of $11.50 \% /$ $11.24 \% / 17.29 \%$ for water and $15.32 \% / 13.96 \% / 32.69 \%$ for microplastics. Roseovarius was the major contributor among the predominant genera for mexW/cpx $\mathrm{R}$ with the proportion of $7.04 \% / 7.04 \%$ for water and $16.00 \% / 16.00 \%$ for microplastics. Ruegeria contributed to occurrence of $m a c \mathrm{~B}, m e x \mathrm{~W}$, tet $\mathrm{B}(\mathrm{P})$, and $c p x \mathrm{R}$ with proportion in the range of $4.12 \%-8.83 \%$ for water and $5.70 \%-9.95 \%$ for microplastics. The predominant genera generally posed more contribution to proliferation of ARGs on surface of microplastics than that in water, illustrating that bacterial community might have more important effect on ARGs at presence of microplastics.

Co-occurrence network was used to investigate the potential correlation among genera and ARGs (Fig. 6(a)). Total 10 genera frequently occurring in samples and 15 ARG subtypes with the relatively high abundance were selected to construct the network among them. ARG subtype and bacterial genus showed different preference according to co-occurrence network analysis. Multidrug resistance gene $e m r$ E was significantly positively related to Mycobacterium, Muricauda, Ruegeria, Roseovarius, and Roseobacter, illustrating that these bacterial genera might possess high potential to host multidrug resistance genes so as to induce high potential health risks for humans. Chloramphenicol resistance gene flo $\mathrm{R}$ which was the subtype with the highest abundance in all samples and 
(a)

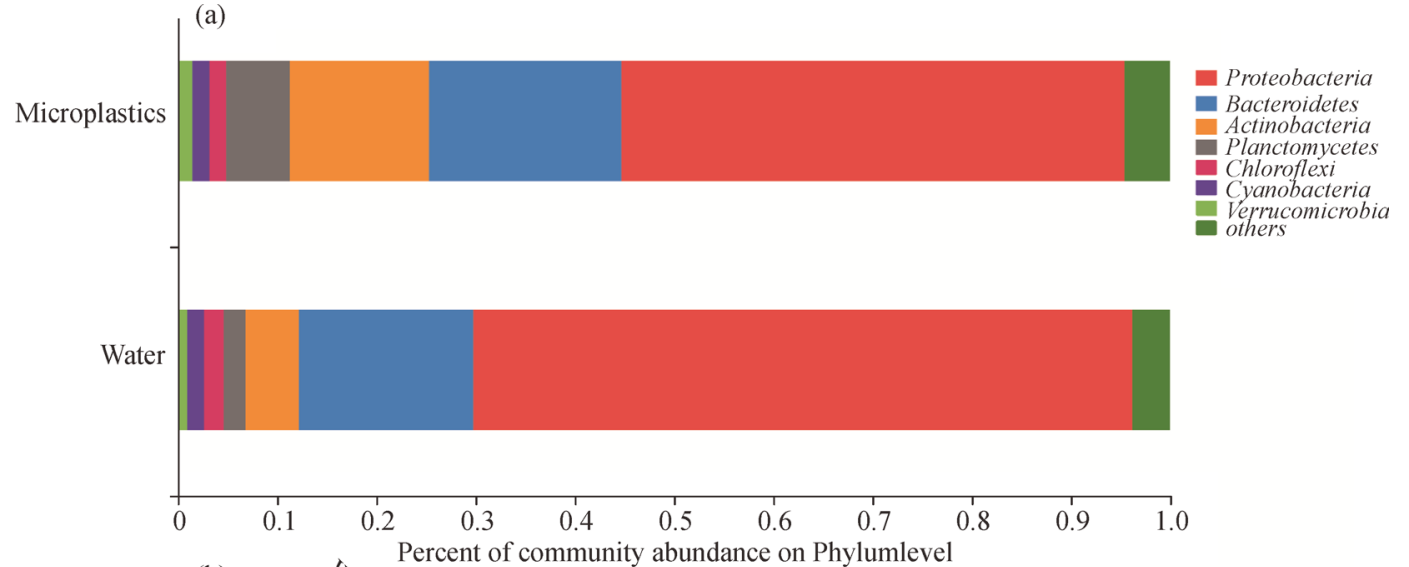

(b)

Percent of community abundance on Phylumlevel

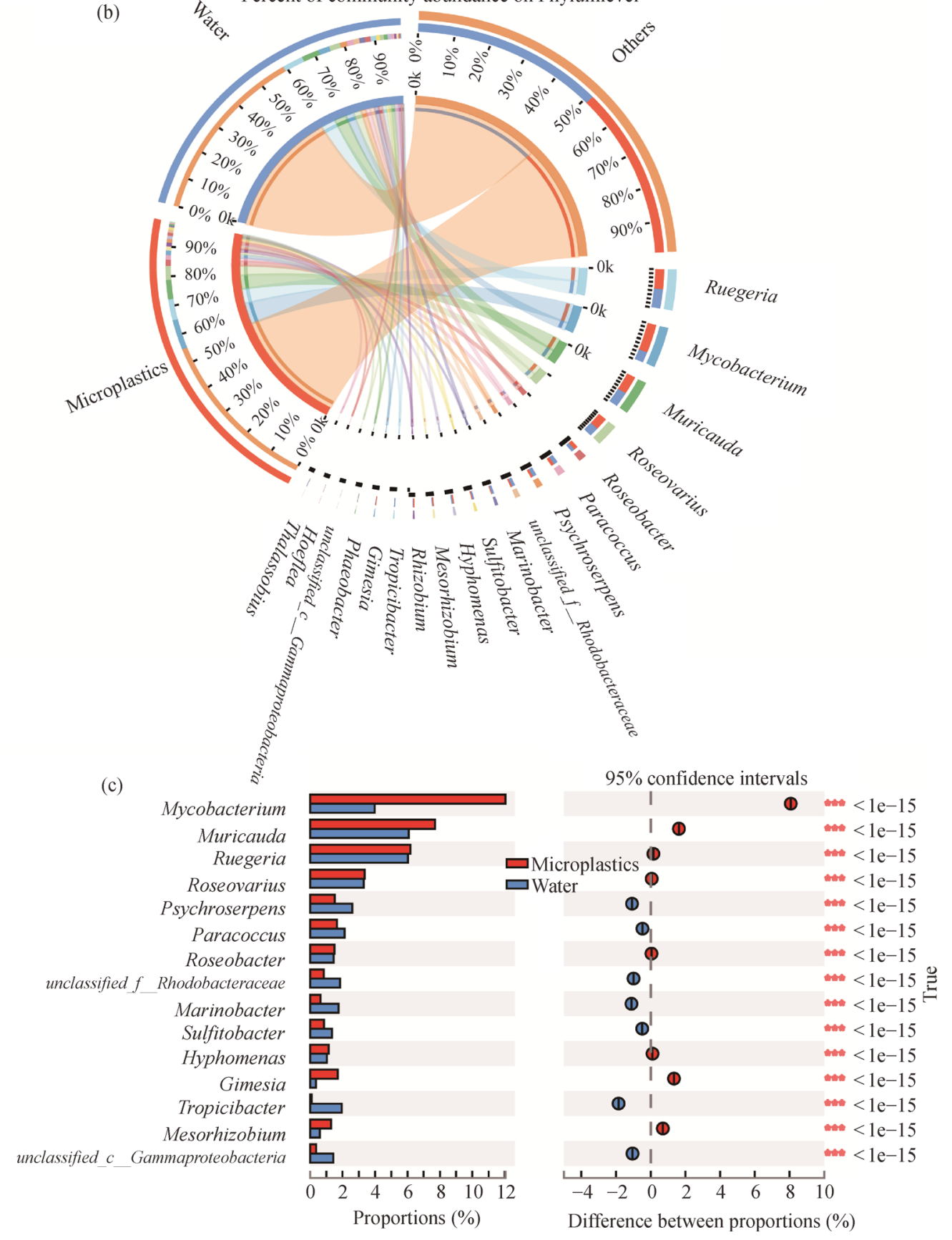

Fig. 3 Bar plot on phylum level (a), Circos diagram on genus level (b), and Fisher's exact test bar plot on phylum level (c) of water and microplastic samples. 


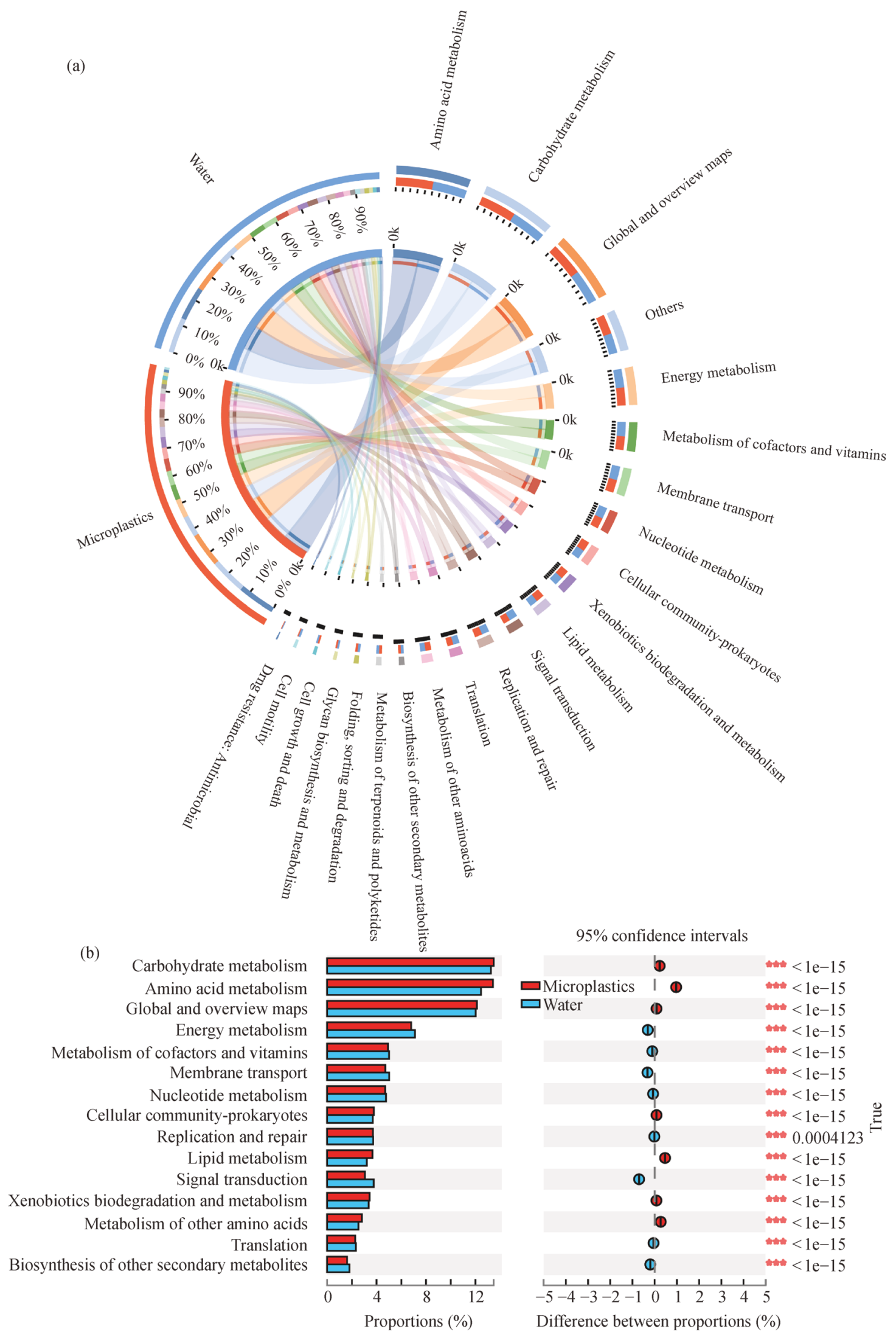

Fig. 4 Circos diagram (a) and Fisher's exact test bar plot (b) of KEGG level 2 pathway in water and microplastic samples. 


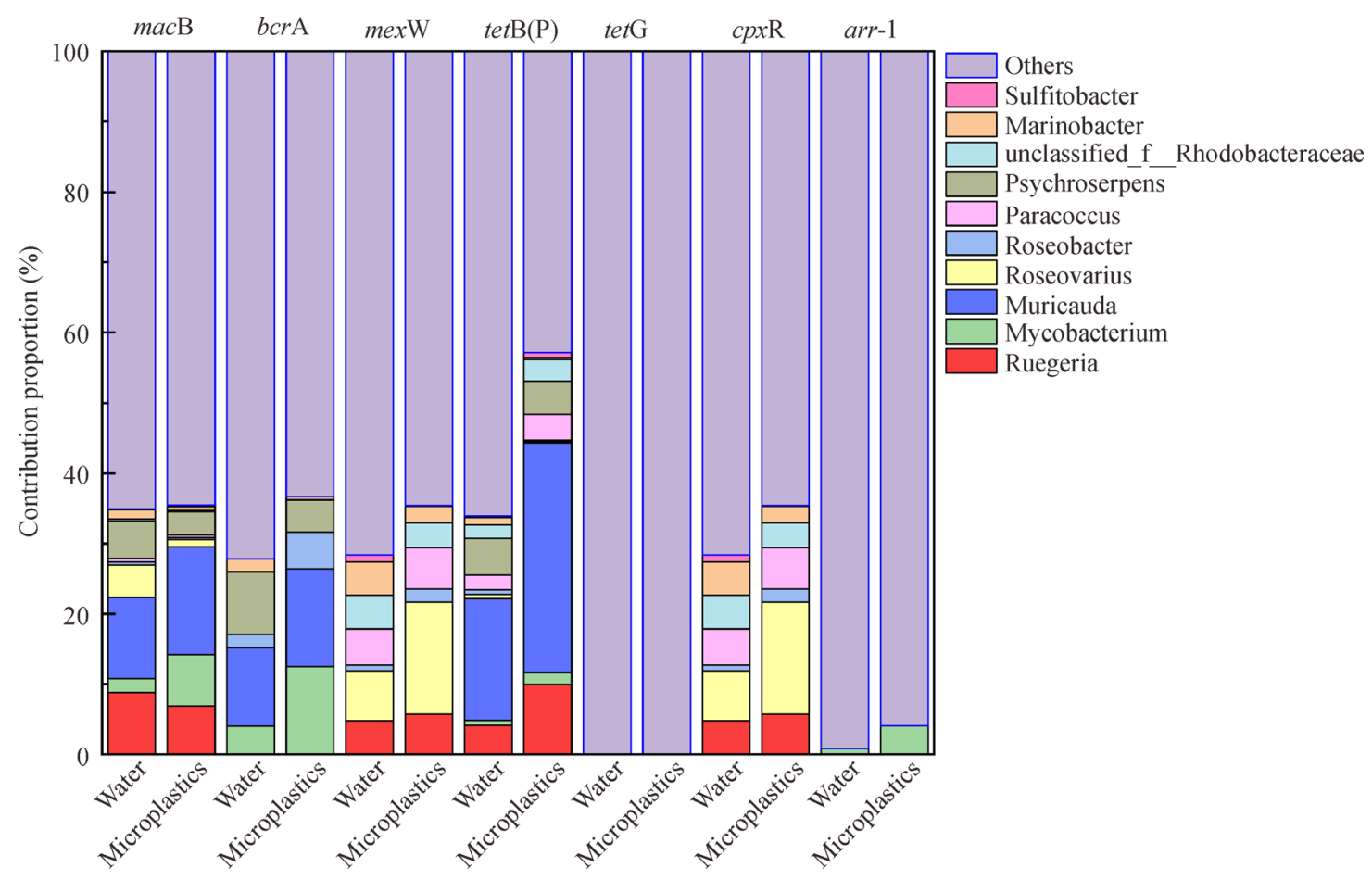

Fig. 5 Contribution of major bacterial genera to proliferation of representative ARGs.

chloramphenicol exporter were significantly positively related to 5 genera including Psychroserpens, Paracoccus, unclassified_f_Rhodobacteraceae, Marinobacter, and Sulfitobacter while another chloramphenicol resistance gene cat $\mathrm{B}$ was positively related to Mycobacterium, Muricauda, Ruegeria, Roseovarius, and Roseobacter. Similar to chloramphenicol resistance genes, tetracycline resistance gene subtypes also showed different relationship with the bacterial genera. Gene tetX was positively related to Mycobacterium, Muricauda, Ruegeria, Roseovarius, and Roseobacter while tet $\mathrm{A}$ and tet $\mathrm{G}$ were positively related to Psychroserpens, Paracoccus, Marinobacter, unclassified_f_Rhodobacteraceae, and Sulfitobacter, exhibiting that ARG subtypes might have different potential of interacting with bacteria. Sulfonamide resistance genes including sul1 and sul2, aminoglycoside resistance genes including $\operatorname{aac}\left(2^{\prime}\right)-\mathrm{I}$, $\operatorname{aph}\left(3^{\prime \prime}\right)-\mathrm{I}, \operatorname{aph}(6)-\mathrm{I}$, and $\operatorname{aadA}$, bacitracin resistance gene $(b a c \mathrm{~A})$, and quinolone resistance gene $(m f p \mathrm{~A})$ were also significantly positively related to 5 genera including Mycobacterium, Muricauda, Ruegeria, Roseovarius, and Roseobacter. Genes including flo R, tet $\mathrm{G}$, tet $\mathrm{A}$, and chloramphenicol exporter were positively related to each other while the remaining 11 ARGs were positively related to each other. Most of ARGs with the relatively high abundance possessed close relationship with both bacterial genera and the other ARG subtypes, suggesting that ARGs in mariculture system containing microplastics might have higher risks to the ecosystem and human health.

Bacterial phylum detected in water and microplastic samples showed wide relation with ARGs (Fig. 6(a)). Three genera of Proteobacteria exhibited close relationship with 11 ARG subtypes while Muricauda belonging to Bacteroidetes were also positively related to 11 ARGs. Attention should also be paid to Mycobacterium which was genus of Actinobacteria phylum and a potential pathogenic bacterium because it was positively related to 11 ARGs with the relatively abundance to become a potential multidrug resistance bacterium.

3.5 Correlation among ARGs, MGEs, and MRGs in mariculture system

Total 15 ARGs with the relatively high abundance were significantly positively related with MGEs (Fig. 6(b)). Gene $f$ loR/tet $\mathrm{G} /$ tet $\mathrm{A} /$ chloramphenicol exporter was positively related with 4 integrons including int $I 1$, int $I 2$, int $I 9$, and groEL/int I1 while the remaining ARGs were positively related with 2 integrases including intIA and orfl intI1. Gene intI 1 was positively related with int 2 , int $I 9$, and groEL/intI 1 while int $I 2$ was positively related with intI9 and groEL/intI1. Moreover, gene intI9 was also positively related with groEL/intI1 while intIA was positively related with orflintI 1 .

Total 14 among 15 ARGs with the relatively high abundance were related with 10 MRGs (Fig. 6(c)). Gene $f l o \mathrm{R} /$ tetA/chloramphenicol exporter was significantly positively related with $\operatorname{cop} \mathrm{R}$ while tet $\mathrm{G}$ was not related with any MRGs. The remaining 11 ARGs were significantly positively related with $9 \mathrm{MRGs}$ including $y b t \mathrm{P}, y b t \mathrm{Q}, y f e \mathrm{~B}$, 

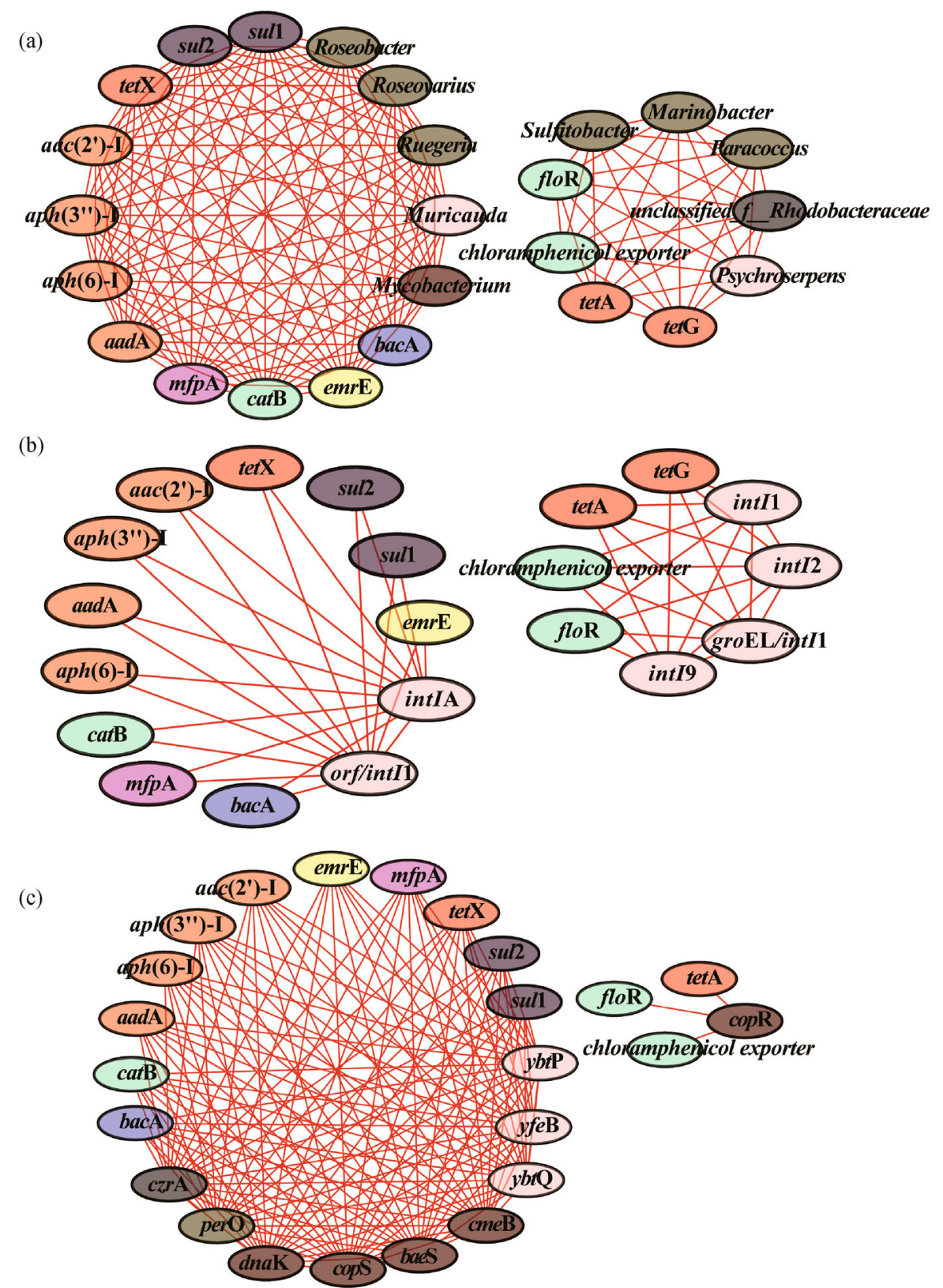

Fig. 6 Co-occurrence network between typical ARGs and bacterial genera (a), ARGs-MGEs (b), and ARGs-MRGs (c). Red lines represent the positive correlation.

$d n a \mathrm{~K}, p e r \mathrm{O}, c o p \mathrm{~S}, b a e \mathrm{~S}, c z r \mathrm{~A}$, and $c m e \mathrm{~B}$. Except copR, the remaining 9 MRGs were positively with each other. Gene tet $\mathrm{X}$ was the most active tetracycline resistance gene to correlate with 4 types of genes resistant to iron, copper, molybdenum, and zinc. Genes resistant to sulfonamides, aminoglycosides, and bacitracin also exhibited close and complex relationship with MRGs.

\section{Discussion}

Resistant antibiotics have been detected in different matrices (Mustafa et al., 2021). Antibiotic chloramphenicol has been banned for food especially animal-food production including aquaculture due to its toxicity although it is still used as human medicine (Hanekamp and Bast, 2015). Chloramphenicol is regarded as important inducer for aplastic anemia and possible carcinogenic agent to exert potential health risks to humans (Hanekamp and Bast, 2015). Previous study reported that chloramphenicol in microbial fermentation food unexpectedly occurred due to possible use of this antibiotic during production (Fraiture et al., 2020). Chloramphenicol resistance genes were also induced due to usage of relative 
antibiotics (Fraiture et al., 2020). Genes such as catB, $c m l \mathrm{~A}$, and $f l o \mathrm{R}$ showed different resistance mechanisms for chloramphenicol antibiotic. Gene $f l o \mathrm{R}$ showed efflux pump conferring antibiotic resistance while $c a t \mathrm{~B}$ and $\mathrm{cmlA}$ were mainly dependent on enzyme inactivation to confer antibiotic resistance. Total 6 among 13 subtypes of chloramphenicol resistance genes were detected with the relatively high abundances in both water and microplastic samples, illustrating chloramphenicol resistance genes might have the relatively strong activity. No chloramphenicol was detected in water sample, illustrating that occurrence and proliferation of chloramphenicol resistance gene might be caused by multiple factors. The abundances of chloramphenicol resistance genes in water or microplastics previously reported (Yang et al., 2019) were much lower than those of this study, illustrating that mariculture environment might be more suitable for proliferation of chloramphenicol resistance genes.

Sulfonamide and tetracycline antibiotics have been widely detected in different matrices (Buta et al., 2021; $\mathrm{Lu}$ et al., 2018; Wen et al., 2021). Sulfonamide and tetracycline resistance genes also frequently proliferated in the environment with the relatively high abundances $(\mathrm{Lu}$ et al., 2019b). Genes including sul 1 and sul2 confer the antibiotic resistance through protein replacement to serve as the predominant sulfonamide resistance gene. The relative abundance of sul 2 in both water and microplastics was lower than that of sul1 reported by this study while relative abundances of sul 1 were lower than those of sul2 in a recirculating aquaculture system although abundances of sul genes in microplastic samples were higher than those in water for both studies (Lu et al., 2019a), which might be affected by environmental conditions of different aquaculture system. Total 21 tetracycline resistance genes were detected in the samples of this study with resistance mechanisms including efflux pump (e.g. tet 35 , tet $\mathrm{A}, \operatorname{tet} \mathrm{G}$, and $t e t \mathrm{~B}$ ), antibiotic target protection protein (e.g. tet32, tet $\mathrm{M}$, and tet $\mathrm{P})$, antibiotic inactivation enzyme (tet $\mathrm{X})$, and molecular bypass (tet34).

Aminoglycoside and multidrug resistance genes also possessed relatively high abundance in mariculture system of this study. Total 40 multidrug resistance subtypes and 18 aminoglycoside resistance subtypes existed in the samples. Resistance mechanism of aminoglycoside resistance genes was mainly antibiotic inactivation enzyme while multidrug resistance mechanism was mainly efflux pump according to ARG annotation. Multidrug resistance genes are generally defined as gene resistant to $\geqq 3$ different classes of antimicrobial agents or gene/mutation/bacterial isolate exhibiting $\geqq 3$ different resistance phenotypes to originate from human, animal, or both human and animal sources (Wendlandt et al., 2015). The relative abundances of multidrug resistance genes on microplastics were higher than those in water, illustrating that microplastics might be potential host to readily concentrate multidrug resistance genes from water to pose higher health risks.
Macrolide-lincosamide-streptogramin and quinolone antibiotics have been frequently detected in aquatic environment so that their corresponding resistance genes have also widely existed in the environments including aquaculture system (Lu et al., 2019a; Wang et al., 2018). Total 13 macrolide-lincosamide-streptogramin resistance gene subtypes and 4 quinolone resistance gene subtypes were detected in the mariculture system. The mechanisms of resistance to macrolide-lincosamide-streptogramin mainly included efflux pump (e.g. oleB and mefA), antibiotic inactivation enzyme (e.g. ere A and $m p h \mathrm{~A}$ ), and antibiotic target modifying enzyme (e.g. erm(38) and erm $\mathrm{F}$ ) while those of quinolone resistance genes comprised antibiotic target protection protein and efflux pump. The relative abundance of erm F/qnrA in water/microplastics of this study was lower than that of previous study while abundance of $q n r \mathrm{~S}$ in water/microplastics of this study was higher than that previously reported (Lu et al., 2019a), illustrating that aquaculture environments might have effect on distribution of ARG subtypes.

MGEs are regarded as the indicator for evaluating mobility of ARGs (Lu et al., 2019a; 2019b). Similar to the previous reports (He et al., 2017; Huang et al., 2017; Zhou et al., 2020), intI genes especially class 1 intergron intI 1 served as the major MGEs in water (Fig. 2(a)). The relative abundance of groEL/int I 1 was also high with the relative abundance of 3.54 copies/16S rRNA copy in water and 3.39 copies/16S rRNA copy on microplastics. The relative abundances of MGEs in water or on microplastics of this study were significantly higher than those previously reported (Subirats et al., 2018; Lu et al., 2019a; 2019b), illustrating that higher mobility potential for ARGs in mariculture system of this study. Relative abundances of intI genes in water were higher than those on microplastics of the mariculture system, which was contrary to the previous report (Lu et al., 2019a) to be possibly affected by the aquatic environment and other factors.

MRGs often co-occur with ARGs in different matrices (Flach et al., 2017; Yang et al., 2019). The relative abundances of MRGs were significantly higher than those of ARGs in this mariculture system, which was similar to pattern previously reported (Flach et al., 2017; Yang et al., 2019). The relative abundances of MRGs in water or microplastics were almost 4 order of magnitudes those in the marine environment (Yang et al., 2019), suggesting that MRGs might be more readily to proliferate in this mariculture system. Moreover, MRGs were readily enrich in microplastics than ARGs in this study. Multidrug resistance gene emrE was positively related to metal resistance genes with high abundances to pose higher risks to humans so as to need more attention.

It still needs to clarify that sample quantity could affect the analysis results to some extent. It is regretful that the influential factors of resistance genes in water and microplastic samples could not be determined by pathway analysis due to the limited samples. The future work 
should pay attention to collection of more samples to obtain useful information on source apportionment of resistance genes.

Microplastics are generally regarded as the reservoir of resistance genes and other emerging contaminants $(\mathrm{Lu}$ et al., 2019a; 2021a; Yang et al., 2019). This study also reported that microplastics could enrich most of MRGs (resistant to arsenic, cadmium, cobalt, copper, iron, molybdenum, nickel, and zinc) and some ARGs including genes resistant to aminoglycoside, bacitracin, fosmidomycin, multidrug, polymyxin, macrolide-lincosamide-streptogramin, quinolone, sulfonamide, and vancomycin antibiotics from water in this mariculture system. ARGs or MRGs frequently proliferated in aquaculture environments (Wang et al., 2018; Wang et al., 2019). Therefore, ARGs generally existed in aquaculture systems with relatively high abundances (Wang et al., 2018; Lu et al., 2019a). Similar to microplastics in the effluents of wastewater treatment plants (Martínez-Campos et al., 2021), the dominant phyla of microplastics in this study also included Proteobacteria, Bacteroidetes, and Actinobacteria. The higher relative abundance of Actinobacteria on microplastics might contribute to difference in distribution of ARGs in water and microplastics to some extent. The environmental conditions, microplastic types, microplastic biofilm, and microbial community in microplastics could induce the occurrence and proliferation of resistance genes on microplastics (Martínez-Campos et al., 2021). Microplastics as potential reservoir of MRGs and ARGs should be paid attention for human health and regional sustainability.

\section{Conclusions}

This study adopted metagenomic analysis to investigate the occurrence of ARGs, MGEs, and MRGs in water and microplastics from a typical mariculture system. Genes resistant to chloramphenicol were the dominant ARGs in samples while genes resistant to copper and iron served as the dominant MRGs. Class 1 intergron int 11 served as the major MGEs while Proteobacteria, Bacteroidetes, and Actinobacteria were the dominant phyla in both water and microplastics samples. ARGs with the relatively high abundances were significantly positively related to the major genera, MGEs, and MRGs. Microplastics might become a potential sink for resistance genes in mariculture system. The findings of this study provide useful information on emerging contaminants such as resistance genes and microplastics in mariculture system to bring some basis for environmental management in the related fields.

Acknowledgements This work was financially supported by the National Natural Science Foundation of China (Grant No. 41877131), Taishan Scholars Program of Shandong Province (No. tsqn201812116), Science and
Technology Service Network Initiative of the Chinese Academy of Sciences (KFJ-STS-QYZX-114), Two-Hundred Talents Plan of Yantai (No. Y739011021), and Wanhua Chemical Group Co. Ltd.

Electronic Supplementary Material Supplementary material is available in the online version of this article at https://doi.org/10.1007/s11783-021$1438-\mathrm{y}$ and is accessible for authorized users.

\section{References}

Al-Salem S M, Uddin S, Al-Yamani F (2020). An assessment of microplastics threat to the marine environment: A short review in context of the Arabian/Persian Gulf. Marine Environmental Research, 159: 104961

Buta M, Hubeny J, Zieliński W, Harnisz M, Korzeniewska E (2021). Sewage sludge in agriculture - the effects of selected chemical pollutants and emerging genetic resistance determinants on the quality of soil and crops: A review. Ecotoxicology and Environmental Safety, 214: 112070

Elizalde-Velázquez G A, Gómez-Oliván L M (2021). Microplastics in aquatic environments: A review on occurrence, distribution, toxic effects, and implications for human health. Science of the Total Environment, 780: 146551

Flach C F, Pal C, Svensson C J, Kristiansson E, Östman M, BengtssonPalme J, Tysklind M, Larsson D G J (2017). Does antifouling paint select for antibiotic resistance? Science of the Total Environment, 590-591: 461-468

Fraiture M A, Deckers M, Papazova N, Roosens N H C (2020). Detection strategy targeting a chloramphenicol resistance gene from genetically modified bacteria in food and feed products. Food Control, 108: 106873

Hanekamp J C, Bast A (2015). Antibiotics exposure and health risks: Chloramphenicol. Environmental Toxicology and Pharmacology, 39 (1): 213-220

He X, Xu Y, Chen J, Ling J, Li Y, Huang L, Zhou X, Zheng L, Xie G (2017). Evolution of corresponding resistance genes in the water of fish tanks with multiple stresses of antibiotics and heavy metals. Water Research, 124: 39-48

Huang L, Xu Y B, Xu J X, Ling J Y, Chen J L, Zhou J L, Zheng L, Du Q $P$ (2017). Antibiotic resistance genes (ARGs) in duck and fish production ponds with integrated or non-integrated mode. Chemosphere, 168: 1107-1114

Koutnik V S, Leonard J, Alkidim S, DePrima F J, Ravi S, Hoek E M V, Mohanty S K (2021). Distribution of microplastics in soil and freshwater environments: Global analysis and framework for transport modeling. Environmental Pollution, 274: 116552

Li L G, Huang Q, Yin X, Zhang T (2020). Source tracking of antibiotic resistance genes in the environment: Challenges, progress, and prospects. Water Research, 185: 116127

Liu X, Wang J (2020). Algae (Raphidocelis subcapitata) mitigate combined toxicity of microplastic and lead on Ceriodaphnia dubia. Frontiers of Environmental Science \& Engineering, 14(6): 97

Lu J, Lin Y, Wu J, Zhang C (2021b). Continental-scale spatial distribution, sources, and health risks of heavy metals in seafood: challenge for the water-food-energy nexus sustainability in coastal 
regions? Environmental Science and Pollution Research International, (In press)

Lu J, Wu J, Wu J, Zhang C, Luo Y (2021a). Adsorption and desorption of steroid hormones by microplastics in seawater. Bulletin of Environmental Contamination and Toxicology, (In press)

Lu J, Wu J, Zhang C, Zhang Y, Lin Y, Luo Y (2018). Occurrence, distribution, and ecological-health risks of selected antibiotics in coastal waters along the coastline of China. Science of the Total Environment, 644: 1469-1476

Lu J, Zhang C, Wu J (2021c). Removal of steroid hormones from mariculture system using seaweed Caulerpa lentillifera. Frontiers of Environmental Science \& Engineering, 16(2): 15

Lu J, Zhang Y, Wu J (2020b). Continental-scale spatio-temporal distribution of antibiotic resistance genes in coastal waters along coastline of China. Chemosphere, 247: 125908

Lu J, Zhang Y, Wu J, Luo Y (2019a). Effects of microplastics on distribution of antibiotic resistance genes in recirculating aquaculture system. Ecotoxicology and Environmental Safety, 184: 109631

Lu J, Zhang Y, Wu J, Wang J, Cai Y (2020a). Fate of antibiotic resistance genes in reclaimed water reuse system with integrated membrane process. Journal of Hazardous Materials, 382: 121025

Lu J, Zhang Y, Wu J, Wang J, Zhang C, Lin Y (2019b). Occurrence and spatial distribution of antibiotic resistance genes in the Bohai Sea and Yellow Sea areas, China. Environmental Pollution, 252(Pt A): 450460

Martínez-Campos S, González-Pleiter M, Fernández-Piñas F, Rosal R, Leganés F (2021). Early and differential bacterial colonization on microplastics deployed into the effluents of wastewater treatment plants. Science of the Total Environment, 757: 143832

Mustafa M, Wang H, Lindberg R H, Fick J, Wang Y, Tysklind M (2021). Identification of resistant pharmaceuticals in ozonation using QSAR modeling and their fate in electro-peroxone process. Frontiers of Environmental Science \& Engineering, 15(5): 106

Nowrotek M, Jałowiecki Ł, Harnisz M, Płaza G A (2019). Culturomics and metagenomics: In understanding of environmental resistome. Frontiers of Environmental Science \& Engineering, 13(3): 40

Pazda M, Kumirska J, Stepnowski P, Mulkiewicz E (2019). Antibiotic resistance genes identified in wastewater treatment plant systems- A review. Science of the Total Environment, 697: 134023

Pham D N, Clark L, Li M (2021). Microplastics as hubs enriching antibiotic-resistant bacteria and pathogens in municipal activated sludge. Journal of Hazardous Materials Letters, 2: 100014

Prata J C, da Costa J P, Lopes I, Andrady A L, Duarte A C, Rocha-Santos $\mathrm{T}$ (2021). A One Health perspective of the impacts of microplastics on animal, human and environmental health. Science of the Total Environment, 777: 146094

Santana-Viera S, Montesdeoca-Esponda S, Guedes-Alonso R, SosaFerrera Z, Santana-Rodríguez J J (2021). Organic pollutants adsorbed on microplastics: Analytical methodologies and occurrence in oceans. Trends in Environmental Analytical Chemistry, 29: e00114
Sherpa M T, Najar I N, Das S, Thakur N (2020). Distribution of antibiotic and metal resistance genes in two glaciers of North Sikkim, India. Ecotoxicology and Environmental Safety, 203: 111037

Subirats J, Timoner X, Sànchez-Melsió A, Balcázar J L, Acuña V, Sabater S, Borrego C M (2018). Emerging contaminants and nutrients synergistically affect the spread of class 1 integron-integrase (int $I 1$ ) and sul 1 genes within stable streambed bacterial communities. Water Research, 138: 77-85

Wang J H, Lu J, Wu J, Zhang Y, Zhang C (2019). Proliferation of antibiotic resistance genes in coastal recirculating mariculture system. Environmental Pollution, 248: 462-470

Wang J H, Lu J, Zhang Y X, Wu J, Luo Y, Liu H (2018). Metagenomic analysis of antibiotic resistance genes in coastal industrial mariculture systems. Bioresource Technology, 253: 235-243

Wen Q, Yang S, Chen Z (2021). Mesophilic and thermophilic anaerobic digestion of swine manure with sulfamethoxazole and norfloxacin: Dynamics of microbial communities and evolution of resistance genes. Frontiers of Environmental Science \& Engineering, 15(5): 94

Wendlandt S, Shen J, Kadlec K, Wang Y, Li B, Zhang W J, Feßler A T, $\mathrm{Wu} \mathrm{C}$, Schwarz S (2015). Multidrug resistance genes in staphylococci from animals that confer resistance to critically and highly important antimicrobial agents in human medicine. Trends in Microbiology, 23(1): 44-54

Wright S L, Ulke J, Font A, Chan K L A, Kelly F J (2020). Atmospheric microplastic deposition in an urban environment and an evaluation of transport. Environment International, 136: 105411

Wu N, Zhang W, Xie S, Zeng M, Liu H, Yang J, Liu X, Yang F (2020). Increasing prevalence of antibiotic resistance genes in manured agricultural soils in northern China. Frontiers of Environmental Science \& Engineering, 14(1): 1

Xu E G, Ren Z J (2021). Preventing masks from becoming the next plastic problem. Frontiers of Environmental Science \& Engineering, 15(6): 125

Yang Y, Liu G, Song W, Ye C, Lin H, Li Z, Liu W (2019). Plastics in the marine environment are reservoirs for antibiotic and metal resistance genes. Environment International, 123: 79-86

Yin X, Deng Y, Ma L, Wang Y, Chan L Y L, Zhang T (2019). Exploration of the antibiotic resistome in a wastewater treatment plant by a nine-year longitudinal metagenomic study. Environment International, 133(Pt B): 105270

Yin X, Jiang X T, Chai B, Li L, Yang Y, Cole J R, Tiedje J M, Zhang T (2018). ARGs-OAP v2.0 with an expanded SARG database and Hidden Markov Models for enhancement characterization and quantification of antibiotic resistance genes in environmental metagenomes. Bioinformatics (Oxford, England), 34(13): 22632270

Zhou M, Xu Y, Ouyang P, Ling J, Cai Q, Du Q, Zheng L (2020). Spread of resistance genes from duck manure to fish intestine in simulated fish-duck pond and the promotion of cefotaxime and As. Science of the Total Environment, 731: 138693 